\title{
Spongy bone deformation mechanisms
}

\section{Experimental and numerical studies}

\author{
Fahmi Chaari — Julien Halgrin — Éric Markiewicz \\ Pascal Drazetic
}

Laboratoire d'Automatique, de Mécanique

et d'Informatique industrielles et Humaines (UMR 8530 CNRS)

Université de Valenciennes et du Hainaut-Cambrésis

Le Mont-Houy, Jonas2

F-59313 Valenciennes cedex 9

fahmi.chaari@univ-valenciennes.fr

ABSTRACT. In order to identify the spongy bone's mechanical behaviour, we performed compression tests on cylindrical samples. Experimental results show important dispersions and an unexpected inverse strain rate dependency at low range of loading velocities. The origin of the dispersions can be attributed to the combination of the architecture effect and the mechanical properties variation of the constitutive material. In order to understand the inverse strain rate sensitivity, we used a controlled constitutive material to build new equivalent samples with the spongy bone's architecture. These samples were subjected to compression tests. Numerical simulations of compression tests on the same architecture have been carried out with FE models built from $\mu$ Ct data. The obtained results are compared in term of final sample shape and the evolution of the compression force.

RÉSUMÉ. Afin d'identifier le comportement mécanique de l'os spongieux, des essais de compression ont été effectués sur des échantillons cylindriques. Les résultats expérimentaux montrent une dispersion importante et mettent en évidence une influence inattendue de la vitesse de sollicitation. Les dispersions observées peuvent être attribuées à une combinaison de l'effet architectural et des propriétés mécaniques du matériau constitutif. Afin de mieux appréhender cet effet de vitesse, des échantillons équivalents ont été construits, par prototypage rapide, avec la même architecture que l'os spongieux. Des essais de compression ont été réalisés sur ces échantillons. Des simulations numériques sur cette même architecture ont été effectuées à l'aide de modèles EF construits à partir de données de micro-tomographie. Les résultats obtenus ont été comparés en termes de cinématique de déformation et de l'évolution de la force de compression.

KEYWORDS: spongy bone, compression tests, finite element simulation.

MOTS-CLÉS : os spongieux, essais de compressions, simulation éléments finis.

DOI:10.3166/EJCM.18.67-79 @ 2009 Lavoisier, Paris

EJCM - 18/2009. Numerical models in biomechanics, pages 67 to 79 


\section{Introduction}

Nowadays, car manufacturers jointly use experimentation as well as numerical simulation to improve human safety during crash events. Such simulations would be built in order to quantify injuries risks during accidents. Bone's mechanical behaviour is one of the needed knowledge in order to carry out accurate numerical simulation of crash events. For the construction of human head finite element models, on which our research activities are focused, the skull can be considered either as only one layer of bone (Zhou et al., 1995), or as three distinct layers (outer table, diploe, inner table) (Canaple et al., 2003; Ruan et al., 1994). Even if a three layers model allow a better prediction of bone injury, previous works [Dellile et al., 2003; Van Rietbergen et al., 1995) show that spongy bone mechanical properties found in literature provide unsatisfying results.

In the skeleton, three kinds of bone exist: the short bone (i.e. vertebra), the long bone (i.e. tibia) and the flat bone (i.e. ribs, skull). Bones are made up of two different types of bone tissue, the compact bone (the cortical bone) and the spongy bone (the trabecular bone). In contrast to the structure of cortical bone, trabecular bone is a complex network of intersecting curved plates and tubes (trabeculae). The size and shape distribution of the trabeculae varies along the skull's thickness. The spaces in and around the trabeculae contain fluids and bone marrow. At the scale of the bone constitutive material, compact and spongy bones are constituted by concentric bone lamellae (osteon) and collagen fibers. The orientation of this ultrastructure leads to an important anisotropy. In our study, we will overpass this anisotropy and consider the trabeculae constitutive material as homogenous one.

Different authors (Follet et al., 2002; Turner et al., 1993) reported an important dispersion on the mechanical properties of the trabeculae. All these variations are usually explained by the inter individuality, by the osseous remodelling and by the extraction location. Mechanical tests on spongy bone samples show that this structure has a typical cellular material behaviour (Fyhrie et al., 1994; Kopperdahl et al., 1998): it absorbs an important energy before the total compaction. Moreover, the mechanical behaviour is largely dependent on the loading direction (Follet, 2002; Bayraktar et al., 2004) and the strain distribution is not homogeneous in the sample thickness. That is why works on spongy bones are undertaken, in order to give a better understanding of the spongy bone's mechanical behaviour at mesoscopic scale and to identify a macroscopic constitutive material law taking into account these variations.

Since it is restricting to perform tests on skull from human donors, the development and validation of our experimental protocol to identify the parameters of mechanical behaviour law of the spongy bone, were first performed on beef ribs. Indeed, the skull and rib are both flat bones, which consist of two blades of cortical bone separated by a layer of spongy bone. This protocol will be later applied on human bone. 
An experimental protocol consisting of compression tests on spongy bone samples at different loading velocities is proposed. In order to overcome architecture variation from a sample to another, equivalent sample with a controlled architecture is build. Compression tests on this equivalent sample made from a viscous-plastic material are carried out. The same architecture is used to build FE models and to analyse mechanical responses and deformation mechanisms.

\section{Experimental characterisation}

We performed experimental tests on fresh samples. The maximum period of time between the animal death and the specimen cut was five days. The ribs were kept refrigerated at $4^{\circ} \mathrm{C}$ and the samples were tested immediately after their preparation. Fifty three cylindrical samples of $10 \mathrm{~mm}$ of diameter and about $8 \mathrm{~mm}$ in thickness were extracted. Ten cubic samples were also used in order to investigate the deformed shape evolution during compression tests thanks to imaging techniques. Detailed procedure for boring the samples can be found in previous publications (Chaari et al., 2007; Baivier et al., 2005). We defatted the samples in order to avoid the fluid (blood and marrow contained in the trabeculae network) contribution in the mechanical response. Special care was ported in order to avoid trabeculae alteration during defatting process.
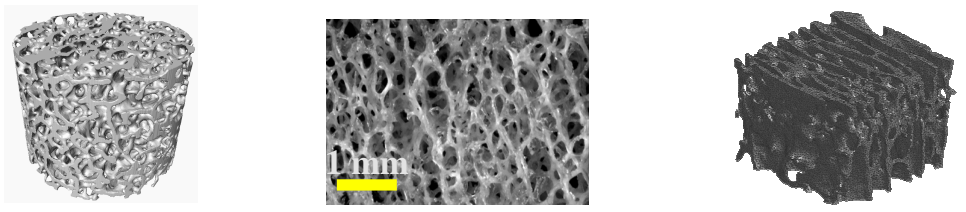

Figure 1. Architectures of a spongy bone samples

In the Figure 1, we can observe the trabeculae network of a defatted spongy bone sample. In addition to the dispersion in the trabeculae and the cells sizes, we can notice a certain orientation in the sample. This is why all the samples have been cut perpendicularly to the rib mean plane.

\subsection{Compression tests}

Compression tests are performed on an electromechanical traction/compression machine at a constant loading velocity. The sample is compressed between two parallel plates.

The typical curve of the compression test can be divided into three stages. At first, the curve shows a quasi-linear compression of the network of trabeculae up to collapse. 
In the second stage, the network of trabeculae crushes until compaction. In the third stage, the total compaction of the sample occurs and the compression force increases quickly. In Figure 2, we plot a typical compression curve obtained for a cubic spongy bone sample. During the compression, one sample side is recorded with a CCD camera. The strain distribution in the sample is homogenous until the peak force. At the second stage of the compression, a shear plane appears in the sample. The third compression stage corresponds to the total compaction of the sample.

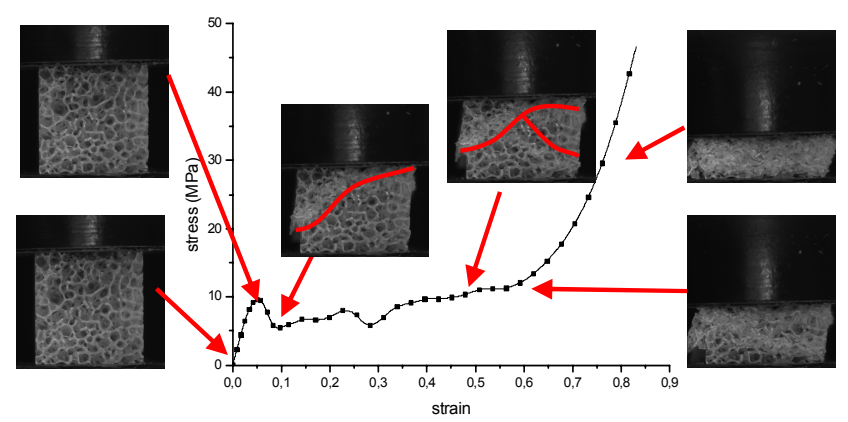

Figure 2. Typical compression curve plotted with the evolution of deformed shape

In Figure 3a, we present the compression curves for 4 samples cut in the same rib and tested at the same velocity. Results are discussed in terms of axial engineering stress and engineering strain. We can also notice that for samples bored from the same rib, the stiffness seems to be constant but the peak value and the transition strains vary significantly. These dispersions are generally attributed to the random architecture, to the strain distribution and to the intrinsic constitutive material variation.
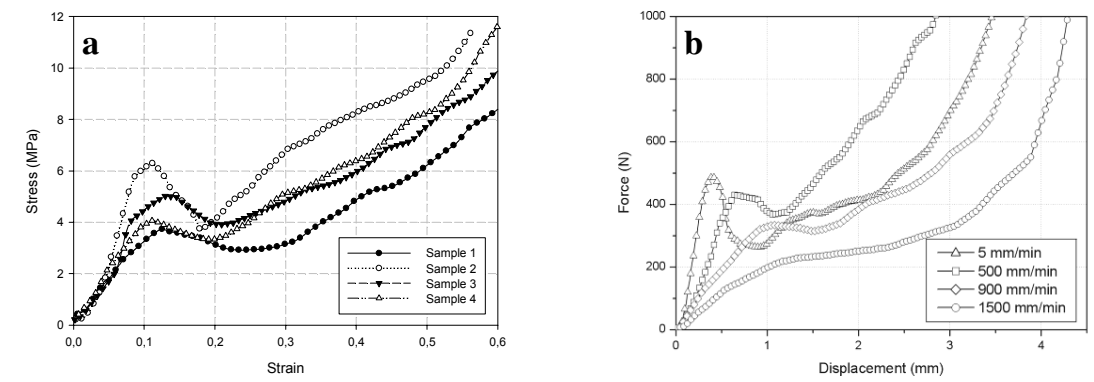

Figure 3. Compression results on different samples cut in the same rib a. loaded at the same velocity $(5 \mathrm{~mm} / \mathrm{min})$, b. loaded at different velocities 
In order to characterize the strain rate effect on the mechanical properties of the spongy bone, samples cut in the same ribs are compressed at different loading velocities.

As shown in Figure 3b, we obtain an unexpected strain rate dependency. When increasing the velocity of loading, the peak force value and the stiffness decrease. All the macro porosity of samples have been defatted, there is no influence of any marrow on the global mechanical behaviour. Microscopic porosities contain fluid which contributes to viscous effects. However, Carter (1977) reported that cortical bone has a classical viscous-plastic strain rate dependency (in the range of 0,001 to $\left.10 \mathrm{~s}^{-1}\right)$. As, the geometrical continuity between the cortical and the spongy bone allow us to suppose that they have the same mechanical properties, this unexpected strain rate sensitivity may also be explained by architecture effects. It is then fundamental to get the real architecture of the spongy bone and check its influence in the global behaviour to compression samples. This investigation has been performed by numerical simulation and the construction of equivalent samples with controlled architecture and constitutive material.

\subsection{Architecture acquisition}

Micro-Computed tomography $(\mu \mathrm{CT})$ was undertaken by an XCT research scanner from StraTek Medizintechnik with a resolution of $20 \mu \mathrm{m}$. For each slice, we obtained a data file containing the space position and the absorption coefficient mean for each voxel. Further details on this technique and its use for bone's characterisation can be found in literature (Follet,2002; Jacobs et al., 1999).

\subsection{Equivalent sample construction}

Thanks to the marching cube algorithm, we reconstructed a surface model of the architecture of some scanned samples of spongy bone. One architecture has been chosen to build physical models by the rapid prototyping technique using a wellknown constitutive material. The ABS polymer is chosen for its classical strain rate dependency. We extracted a cube of $2,7 * 2,7 * 2,7 \mathrm{~mm}$ in the centre of the scanned sample and applied a scale factor of 10 in order to facilitate the strain fields observation. Thanks to this technique, we ensured that the equivalent samples, with the same architecture are identical. Figure 4 presents one equivalent model.

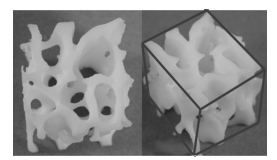

Figure 4. Equivalent cubic sample 


\subsection{Compression test on the equivalent sample}

These equivalent samples were subjected to compression tests between parallel plates using a high speed hydraulic jack and a classical hydraulic machine. The loading velocity range was increased in order to compensate the scale factor effect on the strain rate.

Figure 5a presents the compression results of two equivalent samples loaded at the same velocity $(500 \mathrm{~mm} / \mathrm{min})$. We can notice a good repeatability. This confirms that the dispersions observed on real spongy bone samples can be caused by architecture variability.

In Figure 5a, we can also see that the sample brakes under shear solicitation. This shear phenomenon has also been observed on all the compressed spongy bone samples.
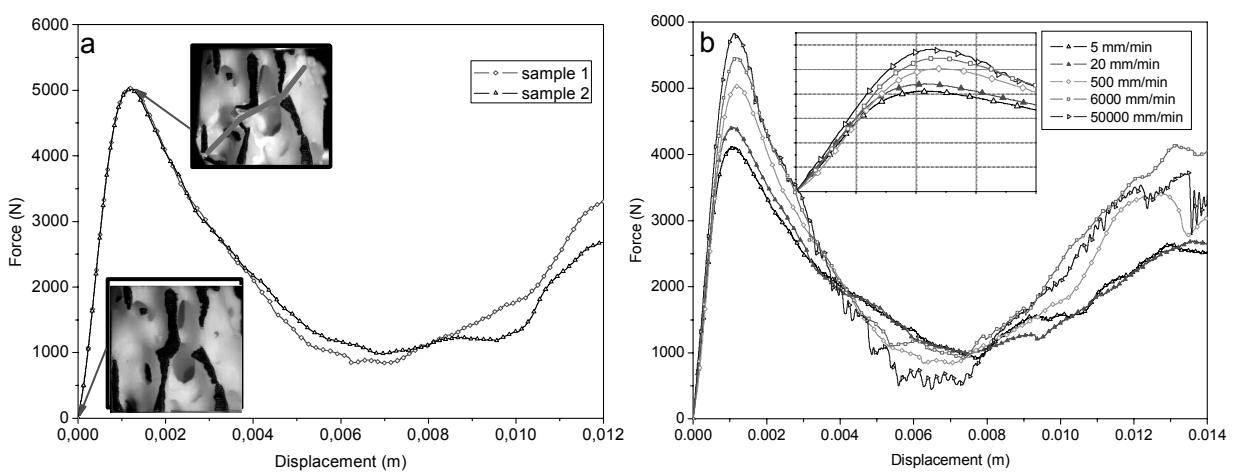

Figure 5. Compression results on equivalent samples a. loaded at the same velocity b. loaded at different velocities

Different loading velocities are applied in order to verify if the architecture is responsible on the unexpected strain rate sensitivity. When we varied the loading velocity (Figure 5b), we obtained a classical strain rate dependency similar to that reported for cellular materials: For higher strain rate in the sample, we obtain a higher peak force. However, the strain rate influence on the stiffness seems to be less significant. These results suggest that the strain rate effect in the spongy bone cannot be explained only by its architecture contribution.

This is why, in order to understand the eventual coupling between architecture and constitutive material effects, we will carry out numerical simulations with the same architecture and different materials behaviours. As a first step of investigation, we will evaluate the efficiency of the FE modelling to reproduce the mechanical response of equivalent samples. 


\section{Numerical study}

Data coming from $\mu \mathrm{CT}$ on the same spongy bone sample have been used to build Finite Element models. First step consisted to apply an adequate threshold in order to distinguish bone parts from to empty volume. The threshold application stage is necessary since $\mu \mathrm{CT}$ data encompasses a wide range of gray levels. We used the Hounsfield absorption coefficient to define the adequate threshold value. This value was adjusted thanks to density measurements that we carried out on the sample before scanning.

According to the element shape, we investigated two ways to build the FE models: Hexahedral or tetrahedral mesh. We built these two models and compared them in term of construction time, geometry accuracy as well as CPU calculation time and results.

\subsection{Hexahedral element models construction}

We wrote a special Matlab ${ }^{\circledR}$ procedure in order to read data from DICOM format and to transform them in hexahedral FE models, known to be adapted to compression solicitation. The easiest way to build cubic elements is to replace each full voxel in every $\mu \mathrm{CT}$ slice, by an element in the same special position. This method gives quite huge models; with more than 10 million elements for a cubic sample for $10 * 10 * 10 \mathrm{~mm}$. Numerical simulation using these models is impossible with our available computing facilities. This is why we decided to group cubic elements in a bigger one. This grouping procedure consists of a 3D scan of all the $\mu \mathrm{CT}$ slices and replaces each 8 neighbour full voxel by a cubic element with the same size. A parametric study was realised in order to validate this grouping procedure. We obtained more suitable FE models, with less and bigger elements. In comparison to the physical model built by Rapid Prototyping, the total mass loss induced by this method in less than $10 \%$ but we admit that some architecture details are not correctly represented. We also programmed connectivity test in order to avoid isolated elements or those who are connected only by one or two nodes to their neighbours.

In order to be closer to experimental conditions, we applied the same scale factor. So, the element size was $0.4 \mathrm{~mm}$. These models are quite easy to simulate but they present a problem of step like in the geometry edge. This problem is inherent to the cubic element shape since they a not able to represent a complex surface.

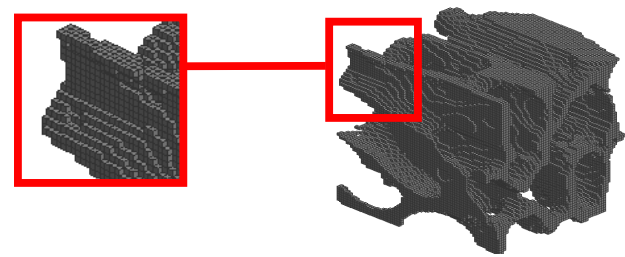

Figure 6. A Hexahedral FE model of a cubic sample with a focus on one trabeculae 
In Figure 6, we present the FE model considered equivalent sample. It is composed of 106000 nodes and 75000 hexahedral elements. The construction of this model lasted 4 hours on a PC. We focus also on one trabeculae in order to emphasis the step like phenomenon. This phenomenon can also affect numerical simulation since the stress is not correctly transmitted to all the structure.

In order to validate our FE models construction method, and to estimate the accuracy of the construction procedure, we used the same data to build a tetrahedral mesh model. These two models will be compared in term of strain distribution and global compression force.

\subsection{Tetrahedral element models construction}

Tetrahedral FE model has been build thanks to the commercial Amira ${ }^{\circledR}$ mesh software. We used the same threshold value and checked the tetrahedral quality to optimise the meshing. The elements construction procedure starts by building surfaces using the marching cube algorithm. The second step is to fill the space between the model surfaces with tetrahedral element.

For the considered equivalent sample, the created Finite Element models contains 31000 nodes and 122000 tetrahedral elements (Figure 7). The construction procedure of this model lasted 1 hours on a PC and the total mass loss induced by this method in less than $5 \%$ in comparison with the physical model. We focus also on one trabeculae in order to show the good representation of all architecture details.

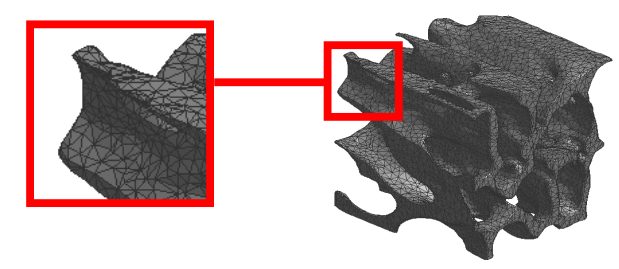

Figure 7. A Tetrahedral FE model of a cubic sample with a focus on one trabeculae

\subsection{Comparison of the mesh quality}

As mentioned before, the constructed models have first been compared in term of construction time and of accuracy. Since Amira ${ }^{\circledR}$ mesh software is optimised for surface generation and elements creation; the construction time for tetrahedral model is clearly lower than our procedure for hexahedral mesh generation.

In comparison with the tetrahedral model, a reduction of $40 \%$ has been reached in terms of elements number. But we have also to notice that the hexahedral meshing 
procedure induced a total volume loss more important than the tetrahedral meshing procedure. This loss can affect the mechanical response during the compression simulation.

Although this comparison shows that the tetrahedral model is more accurate and easier to obtain, it is less adequate for numerical simulation because of the distribution of element size which penalise the time step.

\subsection{Simulation of the compression test on equivalent samples}

\subsubsection{Material law}

Numerical simulation is carried out using an ABS constitutive behaviour law. A bilinear elastic plastic law is used $\left(\mathrm{E}=1100 \mathrm{MPa}, v=0.4, \mathrm{E}_{\mathrm{t}}=100 \mathrm{MPa}\right.$ and $\sigma_{\mathrm{Y}}=40$ $\mathrm{MPa})$. These properties have been identified by tensile testing on plate samples obtained by the same manufactured process.

\subsubsection{Simulation configuration}

Both FE models have been subjected to a similar numerical compression using Pam-Crash $^{\mathrm{TM}}$ explicit finite element code. The choice of this explicit code was motivated by the fact that the ultimate goal of this work is to undertake simulation under dynamic loadings. The compression plates were modelled by two rigid walls. One rigid wall was fixed and the other one had a constant velocity $(6000 \mathrm{~mm} / \mathrm{min})$ with corresponds to the compression speed on equivalent models. The comparison between the ABS numerical models and ABS physical models has been made qualitatively in term of deformed shapes and quantitatively in term of global responses (force/displacement).

\subsubsection{Comparison of $C P U$ times}

The numerical simulation with the hexahedral FE model lasted 21 hours on a PC. The initial time step was 2.12E-06 s. For the tetrahedral FE model, the initial time step was $7.53 \mathrm{E}-09 \mathrm{~s}$. This simulation lasted 290 hours on the same computer. These results confirm the interest of our developed hexahedral mesh FE models building since it reduces the mesh's density and optimizing the computing time.

\subsubsection{Deformed shape evolution}

The comparisons between numerical models and equivalent samples (Figure 8) are in good agreements in terms of global mechanisms. During the compression first stage, we observe a homogeneous strain distribution in the whole sample. Digital image analysis during the experimental compression test showed the same tendency. The structural collapse starts simultaneously for different models. We observe a strain localisation and a shear plane appears in the sample. The location of the shear plane is similar to the experimental one observed on ABS sample. 


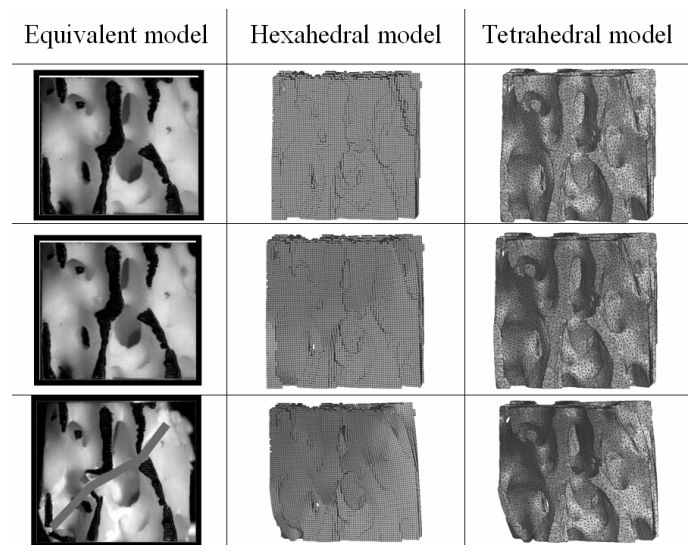

Figure 8. Simulation results for equivalent model and both FE models (nodal displacement evolution)

\subsubsection{Global response comparison}

In Figure 9, we compare global responses of the two FE models to the experimental one for compression velocity of $6000 \mathrm{~mm} / \mathrm{min}$.

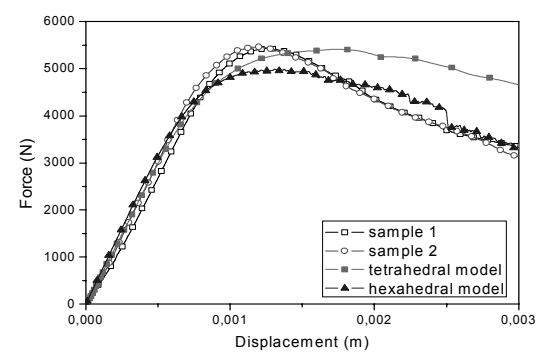

Figure 9. Comparison of numerical and experimental global responses

The stiffness of numerical models is similar to one obtained experimentally, but a significant difference is observed on the peak force value. For the hexahedral model, the difference could be partially explained by the total volume difference. Indeed, as discussed below, hexahedral FE generation induces about $10 \%$ of mass loss. The difference between the tetrahedral and the equivalent model could explain by the formulation of tetrahedral elements in Pamcrash ${ }^{\mathrm{TM}}$. Indeed, the tetrahedral elements do not exist; they are modelled by degenerated hexahedral elements. 


\subsection{Simulation of the compression test on spongy bone}

\section{Simulation configuration}

The numerical simulation conditions are similar to those used for equivalent sample compression. We changed the material constitutive law, the compression velocity $(10 \mathrm{~mm} / \mathrm{min})$, the friction coefficient between sample and the rigid walls $(\mu=0.3)$. An elastic plastic law with parameters reported in previous studies (Chaari et al., 2007; Yu et al., 2005) has been used ( $\mathrm{E}=15000 \mathrm{MPa}, v=0.3, \mathrm{E}_{\mathrm{t}}=49 \mathrm{MPa}$ and $\sigma_{\mathrm{Y}}=200 \mathrm{MPa}$ ). The termination time of the simulation is about $5 \mathrm{~ms}$, which corresponds to a crushing of approximately $10 \%$ of the height sample. Because of scale factor applied on micro CT data for the construction of the equivalent model, the numerical results obtained with bone properties cannot be compared to the experimental ones. We will so limit the comparison to the collapse mechanisms.
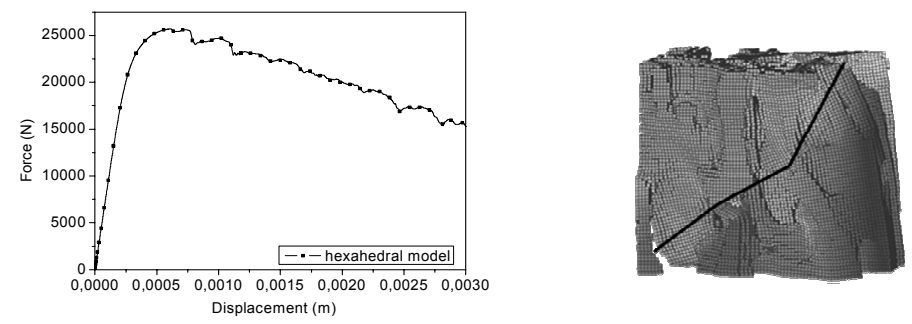

Figure 10. Numerical global responses on spongy bone

In Figure 10, the global response of the spongy bone shows the same collapse phenomenon (shear plane) than the experimental results on equivalent sample.

\section{Discussion and conclusion}

Compression tests on spongy bone samples at different loading velocities showed an unexpected strain rate dependency of this structural material. In order to explain this unexpected behaviour, we launched two ways of investigations: experimental tests on samples with controlled architecture and numerical simulation on FE models with the same geometry.

Compression tests on equivalent samples made of ABS polymer were performed at a wide range of loading velocities. We obtained a classical strain rate dependency with a higher force peak for higher loading velocity. These tests did not explained spongy bone's mechanical behaviour, but they suggested that such behaviour is a combination of the architecture and constitutive material effects at the microscopic scale. 
We carried out numerical simulations of compression tests on the equivalent sample with two different FE models. The construction and the computing time with these hexahedral and tetrahedral models are quite different. Each model presents some advantages but the hexahedral model is a good approximation with a noticeable gain in term of computing time. Despite the difference in global response, these models lead to the same deformation mechanisms. These mechanisms are comparable to those observed during experimental compression tests.

The hexahedral model was used for the simulation of compression test on spongy bone. We obtained also a collapse behaviour, similar to the experimental one. The different on a peak value can be explained by the scale factor applied on the FE model. We notice also that collapse is initiated in the same shear plane for the equivalent samples made of ABS. This observation suggests that the constitutive material effect on the global collapse is limited.

Finally, spongy bone's mechanical behaviour is a complex combination of architecture and constitutive material effects. We investigated one architecture and showed that it is not the origin of the inverse strain rate dependency. Current works are focused on the numerical simulation of different architectures, and viscousplastic behaviour law for trabeculae constitutive material. In order to simulate the entire crushing response, damage and failure criterion will be introduced.

\section{Acknowledgement}

The present research work has been accomplished in the common lab DRSC between the ONERA Lille and LAMIH/CNRS-UVHC. It has been supported by the European Community, the French Ministry for Education, Research and Technology, the Nord-Pas de Calais Region.

\section{References}

Baivier S., Chaari F., Drazetic P. and Markiewicz E., "A micro finite element reconstruction of identifying spongy bone mechanical behaviour”, SB Conference, Brussels, 2005.

Bayraktar H. H. and Keaveny T. M., "Mechanisms of uniformity of yield strains for trabecular bone”, Journal of Biomechanics, 37, 2004, p. 1671-1678.

Canaple B., Rungen P., Drazetic P., Markiewicz E. and Cesari D., "Towards a finite element head model used a head injury predictive tool", Int, Journal of Crashworthiness, 8, 2003, p. 19-30.

Carter D.R. and Hayes W.C., "The compressive behaviour of bone as a two-phase porous structure", Journal of bone and joint surgery, vol. 59, 1977, p. 954-962.

Chaari F., Markiewicz É., Drazetic P. "Identification of the spongy bone mechanical behaviour under compression loads: numerical simulation versus experimental results", International Journal of Crashworthiness vol. 12, $\mathrm{n}^{\circ}$ 3, 2007 p. 247-253. 
Delille C., Baivier S., Masson C. and Drazetic P., "Identification of skull behaviour laws starting from bending tests", Mécanique et Industries, vol. 4, 2003, p. 119-123.

Follet H., Caractérisation Biomécanique et Modélisation 3D par Imagerie X et IRM haute résolution de l'os spongieux humain : Evaluation du risque fracturaire, PHD Thesis, Institut National des Sciences Appliquées de Lyon, 2002.

Fyhrie D. P. and Schaffler M. B., "Failure mechanisms in human vertebral cancellous bone", Bone, vol. 15, 1994, p. 105-109.

Jacobs C.R., Davis B.R., Rieger C.J., Francis J.J., Saad M. and Fyhrie D.P., "The impact of boundary conditions and mesh size on the accuracy of cancellous bone tissue modulus determination using large-scale finite-element modelling", Journal of Biomechanics, 32, 1999, p. 1159-1164.

Kopperdahl D. L. and Keaveny T. M., "Yield strain behaviour of trabecular bone”, Journal of Biomechanics, vol. 31, 1998, p. 601-608.

Ruan J, Awfik T., Khalil T.B., and King A.I., "Dynamic Response of the Human Head Impact by Three-Dimensional Finite Element Analysis", ASME, vol. 16, 1994.

Turner C. H. and Burr D. B., "Basic biomechanical measurements of bone: a tutorial", Bone vol. 14,1993 , p. 595-606.

Van Rietbergen B., Weinans H., Huiskes R. and Odgaard A., "A new method to determine trabecular bone elastic properties and loading using micromechanical finite-element models", Journal of Biomechanics, vol. 28, n 1, 1995, p. 69-81.

Yu H.Y., Cai Z.B., Zhou Z.R. and Zhu M.H., "Fretting behaviour of cortical bone against titanium and its alloy", Wear, vol. 259, n 7-12, 2005, p. 910-918.

Zhou C., Khalil T.B. and King A.I., "A new model Comparing Impact Responses of the Homogeneous and Inhomogeneous Human Brain”, SAE, n952714, 1995, p. 121-137. 
